\title{
Iterative analysis of concrete gravity dam-nonlinear foundation interaction
}

\author{
A. Burman ${ }^{1}$, D. Maity $^{2 *}$, S. Sreedeep ${ }^{3}$ \\ ${ }^{1}$ Dept. of Civil Engineering,BIT Mesra,INDIA \\ ${ }^{2}$ Department of Civil Engineering, IIT Kharagpur, INDIA \\ ${ }^{3}$ Department of Civil Engineering, IIT Guwahati, INDIA \\ *Corresponding author (Email: dmaity@civil.iitkgp.ernet.in)
}

\begin{abstract}
This paper deals with finite element analysis of the soil-structure systems considering the coupled effect of elastic structure and materially nonlinear soil. The equations of motion of both soil and structure have been expressed in terms of displacement variable. The structure and the soil domain are treated as two separate systems. The solution of the coupled system is accomplished by solving the two systems separately and then considering the interaction effects at the soil-structure interface enforced by a developed iterative scheme. Emphasis has been laid on the study of material nonlinearity of the foundation material in the interaction analysis. The results show the pronounced effects of displacements and stresses for the structure when the foundation is assumed to be composed of nonlinear materials. A widely popular model called Duncan-Chang model has been used for representing nonlinear material behavior of soil/rock. In order to represent the semi-infinite nature of the foundation domain, the Lysmer-Kuhlemeyer boundary condition consisting of viscous dashpots have been used. Studies show the accuracy of the proposed algorithm, while comparing with the results reported in the literature.
\end{abstract}

Keywords: Concrete gravity dam; dam-foundation interaction; iterative algorithm; Duncan-Chang model; viscous dashpots.

\section{Introduction}

Considerable research has been conducted on the subject of dynamic analysis of structure-foundation system. The various numerical methods developed for the analysis of dynamic soil-structure interaction (SSI) problems can be classified into two main groups: the direct method and the substructure method. In the direct method, the structure and a finite, bounded soil region adjacent to the structure (near field) are modeled by the standard finite-element method and the effect of the surrounding soil (far field) is analyzed approximately by imposing transmitting boundaries along the near field/far field interface. In the substructure method, the soil-structure system is divided into two substructures. Here, a portion of the soil region may be considered as a part of the structure system. These substructures are connected by the general soil-structure interface and the interaction force for the combined soil-structure interface is calculated considering the contribution of free field analyses and the reaction of the unbounded soil on the general soil-structure interface.

The transmitting boundaries or non reflecting boundary conditions (NRBC) are required if one wishes to use exclusively FEM to simulate the semi- infinite soil region. There are two main types of NRBCs: approximate local NRBCs and exact nonlocal NRBCs. The first local boundary indeed the first transmitting boundary was proposed by Lysmer and Kuhlemeyer (1969). It is also known as viscous or absorbing boundary since it places viscous dashpots at the boundary to absorb the energy of the traveling waves. Zhao and Liu (2003) used operator splitting method to derive an exact NRBC on the truncated boundary of the computational domain in time domain.

Boundary element method has also been widely used to tackle SSI problems. In this method, only the boundaries of the unbounded medium are discretized so that the spatial dimension is reduced by one and the radiation condition is satisfied automatically as a part of the fundamental solution (Zienkiewicz et al., 1977; Estorff and Kausel, 1989). However, it is not very easy to derive the fundamental solutions for many types of problems. Wolf and Song (1996) developed the scaled boundary 
element method (also known as consistent infinitesimal finite element cell method). This method is exact in radial direction and converges to the exact solution in the finite element sense in the circumferential direction.

Bettes (1977) introduced infinite elements, which may be considered to be a natural extension of finite elements to treat infinite domains. Since then, many researchers including Valiappan and Zhao (1992), Khalili et al. (1997) have successfully used infinite elements to model wave propagation problems in infinite domains. This approach requires modeling the near field by FEM and the far field by infinite elements.

Felippa and Park (1980) discussed in detail, staggered solution procedure for a variety of coupled field dynamic problems. Direct solution methods of coupled field problems suffer from the need of excessive storage requirements and computation time. The staggered solution schemes allow us to use reduced matrices for the particular subsystem leading to less time for solution. Maity and Bhattacharya (2003) suggested an iterative scheme in conjunction with the staggered solution procedure for the dam-reservoir interaction problems. However, the staggered approach should be used with great care, since its stability is conditional on the size of time step. If a corrective iteration at each time step is employed, where the interface boundary conditions are iteratively updated until convergence is achieved, one obtains an iterative coupling method. Within the iteration procedure at every time step, a relaxation operator may be applied to the interface boundary conditions in order to enable or speed up convergence. Jahromi et al. (2007, 2009) used partitioned analysis technique involving iterative coupling procedure for solving different soil-structure interaction problems. Degroote et al. (2010) demonstrated that only an approximate Jacobian is needed, as long as it describes the reaction to certain components of the error on the interface's position. Based on this insight, a quasi-Newton coupling algorithm with an approximation for the inverse of the Jacobian (IQN-ILS) has been developed.

Nonlinear SSI analysis for a structure embedded in a half space for a vertical earthquake excitation was carried out by Wolf and Darbre (1986). Wolf and Obernhuber (1985) used indirect boundary element method which involves Green's function in time domain for nonlinear SSI analysis for a surface foundation. Abouseeda and Dakoulas (1998) used a combined FE-BE formulation to represent nonlinear seismic soil-structure interaction in two dimensions in dam-foundation coupled problems. Estorff and Firuziaan (2000) developed a coupled BEM-FEM technique for nonlinear dynamic soil-structure interaction problems.

In this paper, a time domain staggered solution approach with iterative scheme has been used to solve dynamic dam-foundation interaction problems. This method performs all the calculations in time domain in contrast to the most of the substructure techniques. The substructure techniques, while computing the interaction forces require evaluating the convolution integrals which are computationally very demanding and complex processes (Wolf 1985). It doesn't require calculation of the free field motion of the foundation as is required by the substructure method and all the calculations are performed in terms of the absolute displacements. The advantage of this staggered solution procedure is that there is no need to calculate the coupled mass, damping and stiffness matrices which appear in direct coupling equations of interaction problems. Viscous dashpots have been placed at the boundaries of the foundation domain. For characterizing the behaviors of geologic materials, a popular elastic, nonlinear model namely Duncan-Chang (1970) model has been used with satisfactory results.

\section{Theoretical Formulation}

2.1 Modeling of Dam: In the present work, Koyna concrete gravity dam has been chosen as the structure. As the dam is a structure having a much extended length in the longitudinal direction, the state of the stress and the strains inside the dam body can be effectively analyzed through the plane strain idealization. The dam body is considered to be homogenous and isotropic. For plane strain problems, the constitutive matrix $[D]$ expressing the relationship between stress $\{\sigma\}$ and strains $\{\varepsilon\}$ for the dam monolith is:

$$
\{\sigma\}=[D]\{\varepsilon\}
$$

where,

$$
[D]=\frac{E(1-v)}{(1+v)(1-2 v)}\left[\begin{array}{ccc}
1 & \frac{v}{1-v} & 0 \\
\frac{v}{1-v} & 1 & 0 \\
0 & 0 & \frac{1-2 v}{2(1-v)}
\end{array}\right]
$$

Here $E$ and $v$ are the Young's modulus and the Poisson's ratio of the material of the dam body respectively. 
2.2 Modeling of foundation: In the present work, the foundation material is assumed to be of rock in nature. A nonlinear elastic constitutive model has been chosen to simulate the stress vs. strain behavior of soil/rock which is nonlinear in nature. A popular model for geo-materials namely Duncan-Chang (1970) model has been adopted for the purpose of analysis. For this model, the tangent modulus is given by

$$
E_{T}=\left[1-\frac{R_{f}(1-\sin \phi)\left(\sigma_{1}-\sigma_{3}\right)}{2\left(C \cos \phi+\sigma_{3} \sin \phi\right)}\right] E_{i}
$$

Here, $\quad R_{f}=$ Failure ratio.

$C=$ Cohesion .

$\sigma_{1}, \sigma_{3}=$ Major and minor principal stresses respectively.

$\phi=$ Angle of internal friction for soil/rock material.

The failure ratio, $R_{f}$ is defined as

$$
R_{f}=\frac{\left(\sigma_{1}-\sigma_{3}\right)_{f}}{\left(\sigma_{1}-\sigma_{3}\right)_{u l t}}
$$

However, for the present analysis, only the Young's modulus of the rock material was varied according to eq. (3). The Posisson's ratio was kept constant throughout the analysis. The value of failure ratio was taken to be 0.80 (Bose and Das, 1997).

\subsection{Modeling of Reservoir:}

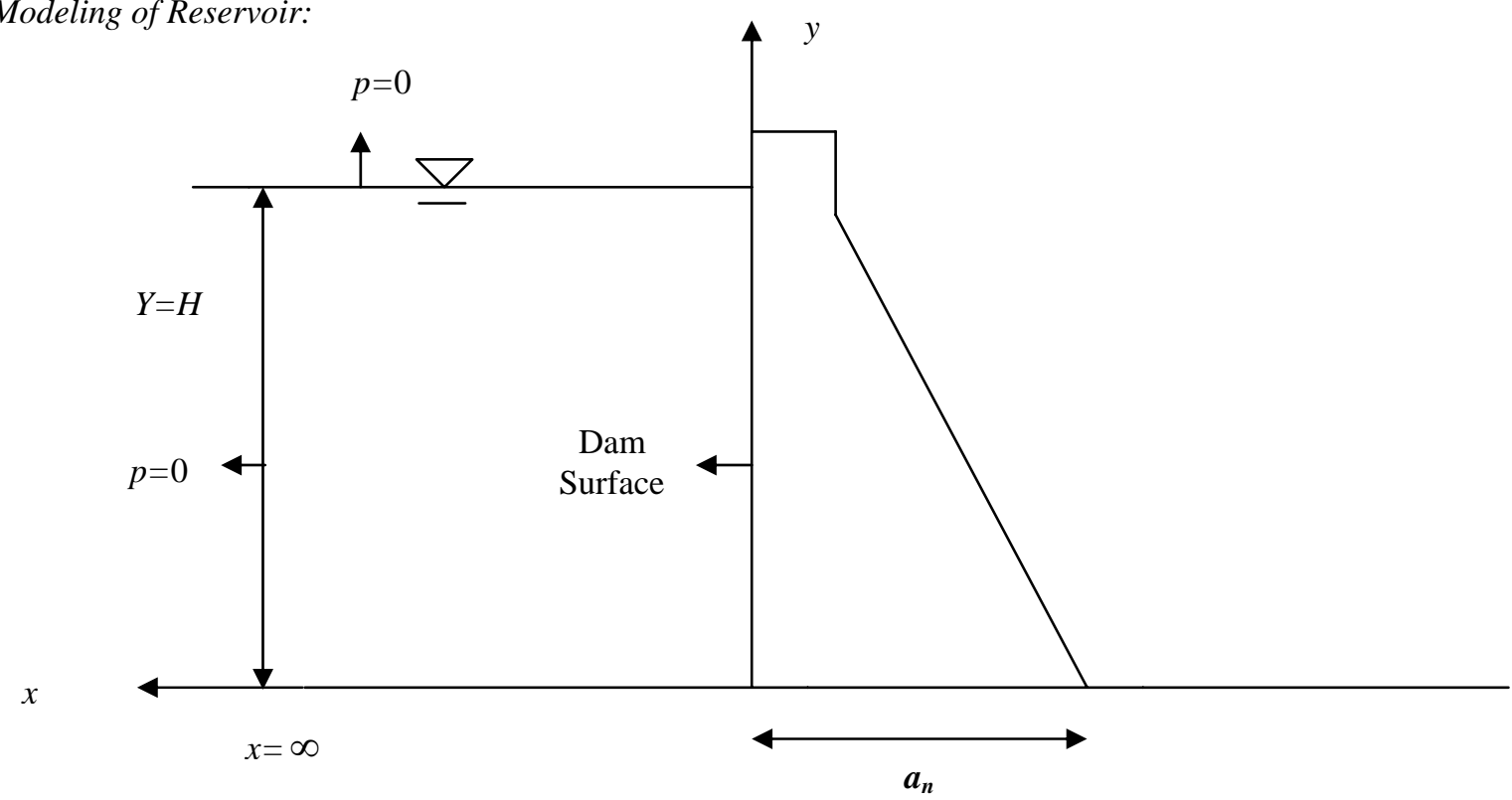

Figure 1. Geometry of the Reservoir Domain

Figure 1 describes the geometry of dam and adjacent reservoir. Assuming the reservoir water to be inviscid and incompressible and its motion to be of small amplitude, the governing equation for hydrodynamic pressure is as follows (Westergaard, 1933):

$$
\nabla^{2} p=0
$$

Here, $\nabla^{2}=\frac{\partial^{2}}{\partial x^{2}}+\frac{\partial^{2}}{\partial y^{2}}$ and is called the Laplacian operator and $p$ is the hydrodynamic pressure. The solution of Laplace equation (eq. 5) can be expressed in eq. (6) with the following assumptions:

i. The bottom of the fluid domain is horizontal and rigid.

ii. The fluid-structure interface is vertical.

iii. The fluid domain extends to infinity and its motion is two dimensional. 


$$
p=2 a_{n} \rho H \sum_{n=1}^{\infty} \frac{(-1)^{n+1}}{\lambda_{n}^{2}} e^{\left(-\lambda_{n} \frac{x}{H}\right)} \cos \left(\lambda_{n} \frac{y}{H}\right)
$$

$p=$ Hydrodynamic pressure at the upstream face of the dam.

$a_{n}=$ The magnitude of acceleration normal to the upstream face of the dam.

$\rho=$ The mass density of water.

$H=$ The height of water at the upstream side of the dam.

$y=$ The variation of distance in the vertical direction.

where, $\quad \lambda_{n}=\frac{(2 n-1) \pi}{2}$

The hydrodynamic pressure on the dam has been calculated using eq. (6).

2.4 Viscous or absorbing boundary: A way to eliminate waves propagating outward from the structure is to use Lysmer boundaries. This method consists of simply connecting dashpots to all degrees of freedom of the boundary nodes and fixing them on the other end (Figure 2). Lysmer boundaries are derived for an elastic wave propagation problem in a one-dimensional semiinfinite bar. The damping coefficient $C_{x}$ of the dash pot equals

$$
C_{x}=A \rho c
$$

where $A$ is the cross sectional area of the bar, $\rho$ is the mass density and $C$ the wave velocity that has to be selected according to the type of wave that has to be absorbed (shear wave velocity $C_{s}$ or compressional wave velocity $C_{p}$ ). In two dimensions, eq. (8) takes the following form, which results in dampers coefficients $C_{n}$ and $C_{t}$ in the normal and tangential directions.

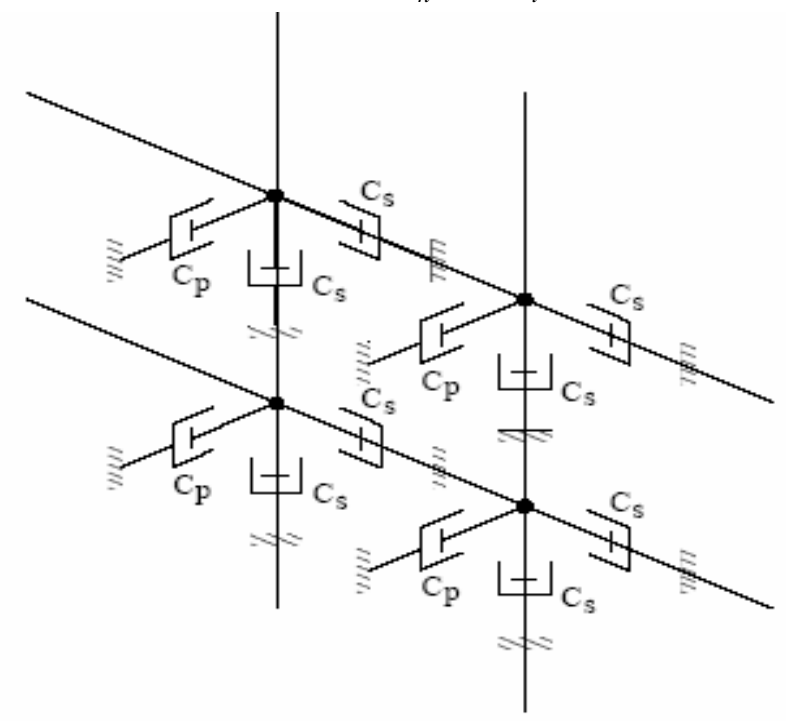

Figure 2. Viscous dashpots connected to each degrees of freedom of a boundary node

$$
\begin{aligned}
& C_{n}=A_{1} \rho c_{p} \\
& C_{t}=A_{2} \rho c_{s}
\end{aligned}
$$

The shear wave velocity $C_{s}$ and compressional wave velocity $C_{p}$ is given by

$$
\begin{aligned}
& c_{s}=\sqrt{\frac{G}{\rho}} \\
& c_{p}=\sqrt{\frac{E(1-v)}{(1+v)(1-2 v) \rho}}
\end{aligned}
$$

Where $G$ is the shear modulus of the medium and is expressed as 


$$
G=\frac{E}{2(1+v)}
$$

Here $E$ is the Young's modulus and $v$ is the Poisson's ratio. This procedure leads to the frequency independent boundary conditions that are local in time and space. If the shape functions of the neighboring finite elements are used instead of crude lumping procedure, a narrow banded damping matrix arises, which is also easy to implement.

However, in general, the directions of the incident waves are not known in advance. In these cases, it may be advantageous to use a 'diffused' version of expression (9) and (10) as suggested by White et al. (1977). Assuming that wave energy arrives at the boundary with equal probability from all directions, effective factors $A_{1}$ and $A_{2}$ are evaluated by minimizing the ratio between the reflected energy and the incident energy over a range of incident angles. For an isotropic medium this results in

$$
\begin{aligned}
& A_{1}=\frac{8}{15 \pi}\left(5+2 S-2 S^{2}\right) \\
& A_{2}=\frac{8}{15 \pi}(3+2 S)
\end{aligned}
$$

which gives slightly better over-all efficiency than the original approach (Lysmer and Kulhemeyer, 1969). The value of $S$ in eq. (14) and eq. (15) is given by

$$
S=\sqrt{\frac{(1-2 v)}{2(1-v)}}
$$

These local viscous dampers represent the exact solution for $P$ - and $S$ - waves which impinge at a right angle on the artificial boundary. They are approximate for inclined body waves, whereby the reflected energy is only a small part of the total energy (Lysmer and Kuhlemeyer, 1969). In many cases, the farther one chooses the artificial boundary to be from a source which radiates waves, the more the angle of incidence with respect to the artificial boundary will approach $90^{\circ}$. In this way, the viscous dampers will perform better. The same applies as the frequency of excitation becomes higher.

2.4 Iterative scheme for SSI problems: The equations of motion for dam and foundation system are written separately as follows:

$$
\begin{aligned}
& M_{d} \ddot{x}_{d}+C_{d} \dot{x}_{d}+K_{d} x_{d}=f_{d} \\
& M_{f} \ddot{x}_{f}+C_{f} \dot{x}_{f}+K_{f} x_{f}=f_{f}+f_{i f}
\end{aligned}
$$

Here, $M, C, K$ and $f$ are the mass, damping, stiffness and the applied load matrices respectively. The suffixes $d$ and $f$ represent dam and foundation domain respectively. The load vector $f_{d}$ includes the hydrodynamic forces developed from reservoir water, the earthquake forces, self weight of the dam body and any other external forces if present. The vector $f_{\text {if }}$ is the vector of interactive force for the soil region exerted by the dam body. The vector $f_{\text {if }}$ is generated during successive iteration at the interface nodes of dam and foundation. The vectors of acceleration, velocity and displacement for the dam part at any time step $t$ are represented by $\ddot{x}_{d}, \dot{x}_{d}$ and $x_{d}$. The vectors $\ddot{x}_{f}, \dot{x}_{f}$ and $x_{f}$ also carry similar meaning for the foundation part.

The damping matrix $C$ has been assumed to be the Rayleigh damping matrix and is constructed as a linear combination of $M$ and $K$ matrices as follows:

$$
C=\alpha M+\beta K
$$

The Rayleigh damping parameters $\alpha$ and $\beta$ are calculated from the following formulae:

$$
\alpha=\frac{2 \omega}{\omega+\omega_{n}} \quad \text { and } \quad \beta=\frac{2 \omega \omega_{n}}{\omega+\omega_{n}}
$$

Where $\omega=$ frequency of earthquake excitation and $\omega_{n}=$ natural frequency of the structure.

The matrices $M_{d}, M_{f}$ and $K_{d}, K_{f}$ are built from the following formulae:

$$
M_{d}=\int_{\Omega_{d}}\left[N_{d}\right]^{T} \rho_{d}\left[N_{d}\right] d \Omega_{d}
$$




$$
\begin{aligned}
& M_{f}=\int_{\Omega_{f}}\left[N_{f}\right]^{T} \rho_{f}\left[N_{f}\right] d \Omega_{f} \\
& K_{d}=\int_{\Omega_{d}}\left[B_{d}\right]^{T}\left[D_{d}\right]\left[B_{d}\right] d \Omega_{d} \\
& K_{f}=\int_{\Omega_{f}}\left[B_{f}\right]^{T}\left[D_{f}\right]\left[B_{f}\right] d \Omega_{f}
\end{aligned}
$$

Where $\left[B_{d}\right],\left\lfloor B_{f}\right\rfloor$ are the strain displacement matrices and $\left[N_{d}\right],\left\lfloor N_{f}\right\rfloor$ are the matrices for the shape functions for dam and the soil foundation portions respectively. Also $\left[D_{d}\right],\left[D_{f}\right\rfloor$ are the constitutive matrices and $\rho_{d}, \rho_{f}$ represent the material densities for dam and foundation respectively.

The iterative scheme has been developed to determine the responses of the dam-foundation coupled system (Figure 3). At any instant of time $t$, the equation of motion for the dam part eq. (17) is solved first with the applied load $f_{d}$ considering dam to be fixed at the bottom. The exerted forces give rise to reaction forces at the common nodes of dam-foundation interface. The reaction forces generated at the common interface nodes of structure and foundation are then applied in the opposite direction at the common nodes of the foundation system to solve eq. (18) at the same time instant $t$. These reaction forces applied in the opposite direction for the foundation part is termed as $f_{\text {if }}$.

After solving the foundation part against the applied load of $\left(f_{f}+f_{i f}\right)$, the common interface nodes for the foundation part will undergo some displacement. These displacements are then fed into eq. (17) i.e. the structural analyzer (eq. 17) in the next instant of time $(t+\Delta t)$ or $t_{i+1}$ as the known displacements at the common interface nodes of dam and foundation. Subsequently, the response of the dam needs to be solved again with the changed boundary conditions which will be different from the earlier step. In this way iterations are continued for a particular time step $t$ until the displacements and stresses for both the dam and foundation part are found to be converged with a certain level of tolerance. The displacements $(x)$ of both the domain at a particular time step are assumed to be converged if the following relationships are satisfied:

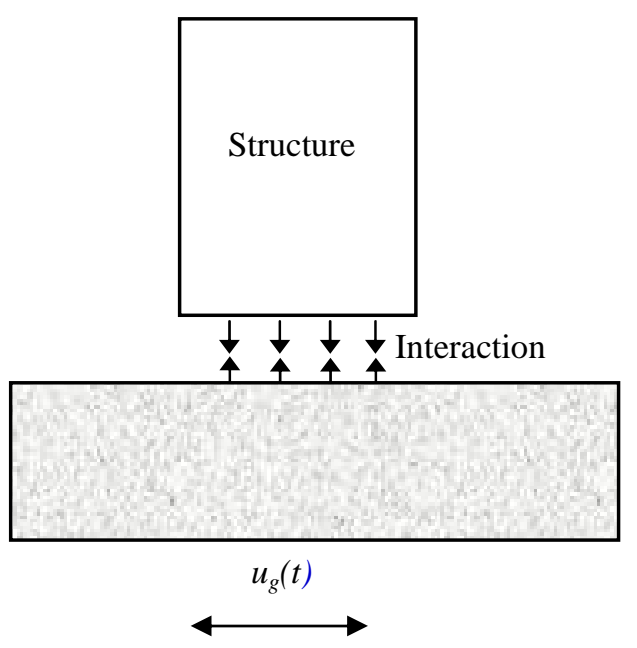

Figure 3. Soil structure interaction employed through reaction forces at the interface

$$
\frac{\left|\left\{x_{d}\right\}_{i+1}^{t}-\left\{x_{d}\right\}_{i}^{t}\right|}{\left|\left\{x_{d}\right\}_{i}^{t}\right|} \leq \varepsilon \quad \text { and } \quad \frac{\left|\left\{x_{f}\right\}_{i+1}^{t}-\left\{x_{f}\right\}_{i}^{t}\right|}{\left|\left\{x_{f}\right\}_{i}^{t}\right|} \leq \varepsilon
$$

Here, $i$ is the number of iteration, $\varepsilon$ is a small pre-assigned tolerance value and $t$ is a particular time instant. Thus this iteration process goes on until the displacements and stresses between two successive iterations converge in both the dam and foundation domain. The value of $\varepsilon$ used in the present analysis is 0.01 . 


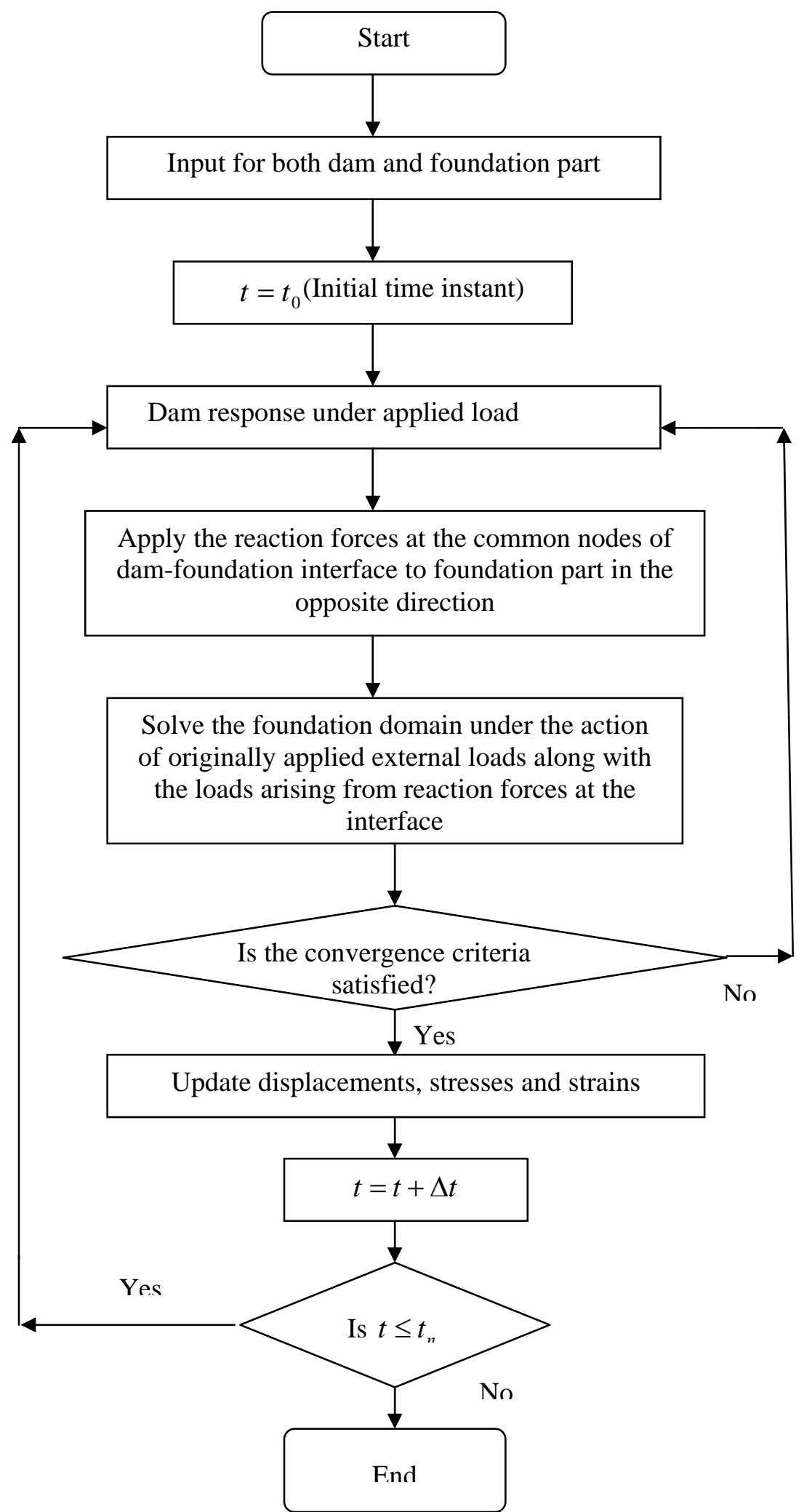

Figure 4. Flowchart of the proposed algorithm for dam-foundation interaction analysis 
A flow chart of the iterative scheme for the solution method of coupled dam-foundation system is presented in Figure 4. In the flowchart, $t_{0}$ stands for the initial time step and $t_{n}$ stands for the final time step. The term $\Delta t$ represents the time step of the implicit-explicit Newmark's method used for the numerical integration of the dynamic equation of motion. The most costly operation involved in the above algorithm is to successively solve two separate systems of equations at each iteration. But in the present case, matrices involved in the solution of the system equations are decomposed into triangular forms at the beginning of the iteration, and thereby only two forward-eliminations and backward substitutions are required at each iteration step. In this way, the time required to obtain the coupled response for a particular time instant is minimized in the developed iterative scheme.

\section{Numerical Results}

3.1 Validation of proposed algorithm: In order to validate the proposed algorithm, a concrete gravity dam -foundation prototype resting on rock foundation was analyzed. The same dam-foundation interaction problem has been previously analyzed by Yazdchi et al (1999). While solving the dam-foundation interaction problem, the side nodes of the discretized finite elements in the foundation portions were considered to be rollers allowing only the horizontal movements and the bottom nodes were kept fixed. The dam and the foundation domain has been discretized using two-dimensional, plane strain, isoparametric finite elements. A $2 \times 2$ Gauss Integration rule was adopted for the calculation of both the stiffness matrix and the mass matrix. The dimensions of the dam prototype are depicted in Figure 5. The width of the base of the dam is $10.0 \mathrm{~m}$. The height of the dam is $15.0 \mathrm{~m}$ out of which the crest portion is of $6.0 \mathrm{~m}$ in length. The width of the crest is considered as $2.0 \mathrm{~m}$. The width and the depth of the foundation part are considered as $100.0 \mathrm{~m}$ and $50.0 \mathrm{~m}$ respectively. The width and height of the foundation domain considered for the analysis purpose are same as those considered by Yazdchi et al (1999). The material properties of the dam and the foundation part are taken same and are as follows:

$\begin{array}{lll}\text { The Young's modulus } & : & 3 \times 10^{7} \mathrm{kN} / \mathrm{m}^{2} \\ \text { The Poisson's ratio } & : & 0.20 \\ \text { The mass density : } & : & 2600.0 \mathrm{~kg} / \mathrm{m}^{3}\end{array}$

3.2 Selection of optimum mesh size: To check the convergence of the results obtained for various mesh grading, a simplified section of the dam prototype (solved by Yazdchi et. al., 1999) has been chosen for the extensive analysis using finite element technique. The slope on the downstream face above the neck of the dam is also neglected in the present analysis. The dimension and the material properties of the dam in the present case are same as listed in section 3.1. The dam is discretized with 8-noded quadratic elements as shown in Figure 5 and is analyzed using plain strain formulation. A concentrated horizontal load of $1000 \mathrm{kN}$ is applied at the crest of the structure and the static analysis is carried out considering the bottom nodes of the dam to be fixed. The structure is discretized with different mesh gradings and the convergences of results for the time periods and the crest displacements obtained for different discretizations. It is observed from the results that the solution converges sufficiently for a discretization of $7 \times 4$ (vertical $\times$ horizontal) mesh grading. But a higher mesh grading $10 \times 4$ was adopted for the analysis for the sake of increased accuracy.

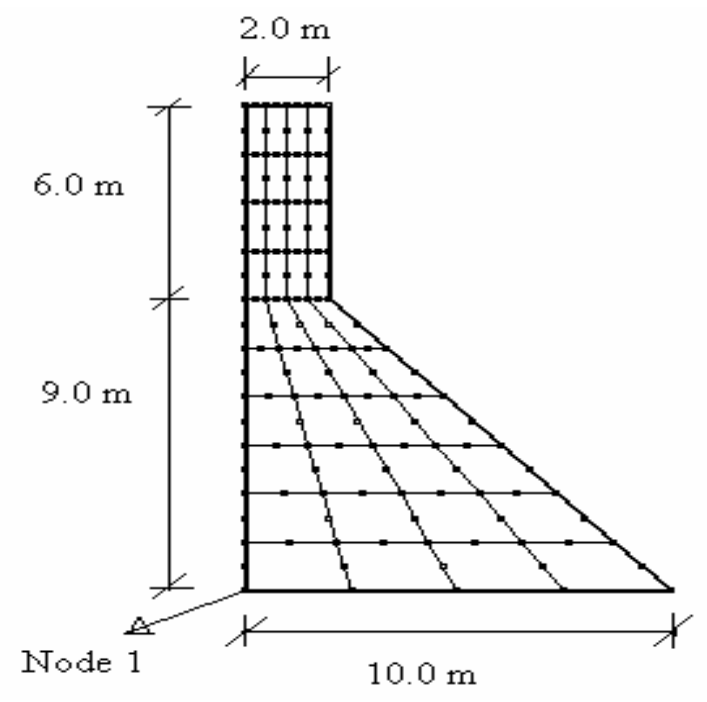

Figure 5. The geometry of dam (Yazdchi et al, 1999) 
For the foundation domain also, similar convergence analyses for mesh grading were carried out. A $1000 \mathrm{kN}$ load was applied vertically downwards at middle of the surface of the foundation domain and static analysis was carried out. The width and the depth of the foundation part are considered to be $100.0 \mathrm{~m}$ and $50.0 \mathrm{~m}$ respectively. The bottom nodes were considered to be fixed in both directions. The side nodes at the truncated boundary were considered to be rollers allowing horizontal movements. The dashpots were modeled as described in section 2.4. It is observed that the result converges for a mesh grading of $12 \times 4$.

3.3 Comparison of proposed model with published results: Having arrived at a suitable mesh grading for both the dam and the foundation domain, an attempt was made to compare the results obtained using the proposed algorithm with the dam-foundation interaction problem solved by Yazdchi et al. (1999). While doing so, suitable boundary conditions were applied at the truncated boundaries as well as at the bottom of the foundation domain and all the concerned nodes were fitted with dashpots. The Koyna earthquake acceleration is applied to the dam prototype with a scaling factor of 2.5. The earthquake acceleration data is presented in Figure 6. Yazdchi et al. (1999) solved this problem by the coupled FEM-BEM method. They included the effect of viscous damping with a damping ratio of 0.05 . Moreover, the effect of hydrodynamic pressure was incorporated in the analysis by the added mass concept proposed by Westergaard (1933). The effect of wave scattering and reflection was tackled by coupled FEMBEM method. When the same dam-foundation model is solved by the proposed iterative scheme in this paper, the effect of viscous damping and the hydrodynamic pressure is also considered in similar way. Also, initially the dam has been analyzed considering the effects of its self weight and the hydrostatic pressure which produced initial acceleration in the dam body. The horizontal extent of the foundation portion is arbitrarily truncated at a certain distance from both the upstream and the downstream face of the dam. The value of tolerance $\varepsilon$ used in eq. 25 is 0.01 . Also, the time interval used for solution of dynamic equilibrium equations (eq. 17 and 18) is 0.01 second.

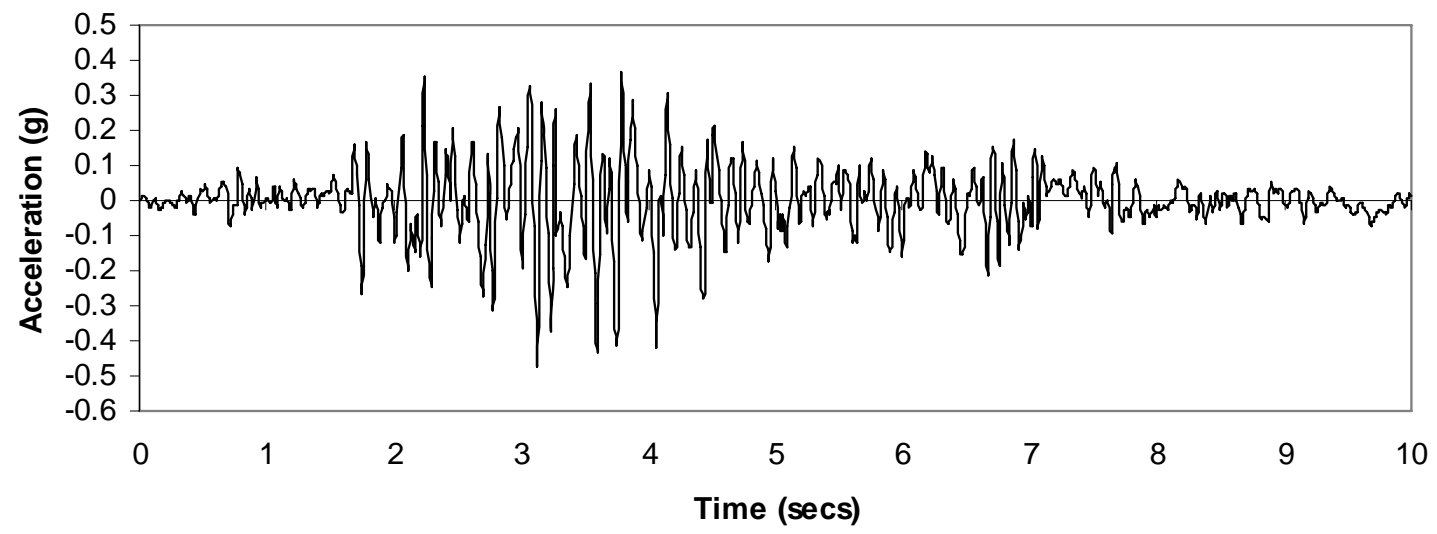

Figure 6. Koyna earthquake acceleration data

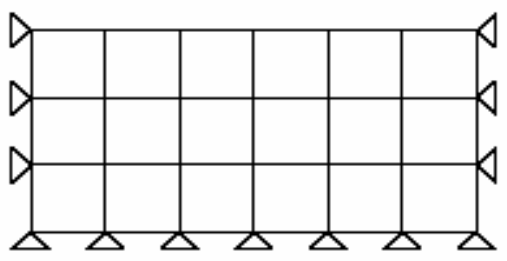

a)

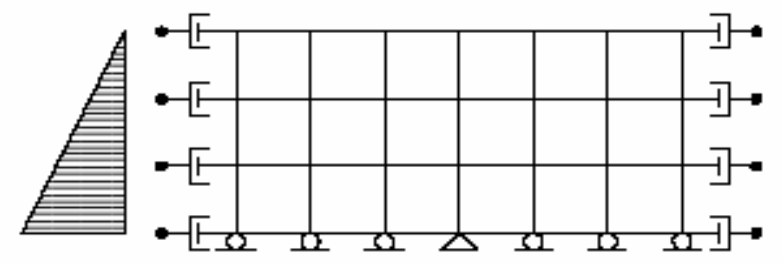

b)

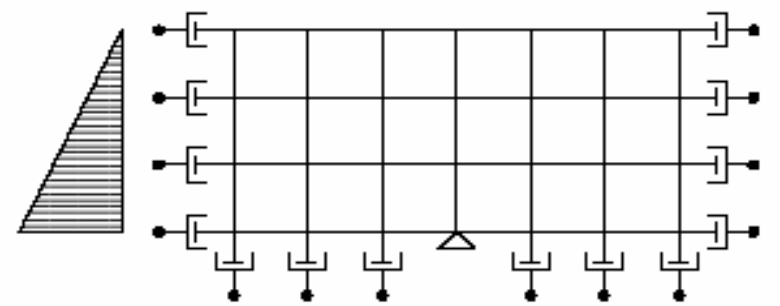

c)
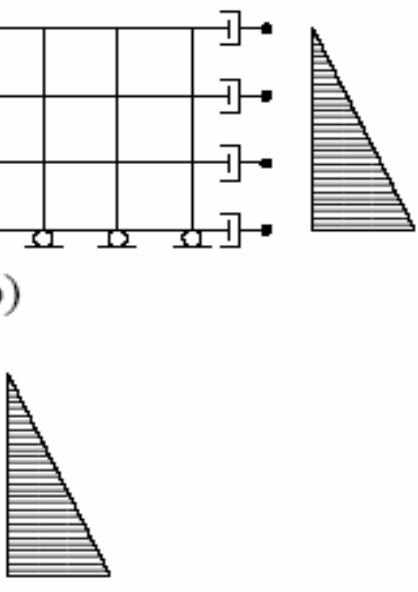

Figure 7. Boundary conditions for 2-D model 
Different boundary conditions were tested on the dam-foundation coupled model. Firstly, the side boundary nodes are fully fixed as shown in Figure 7 (a). Then, these nodes are released and fitted with dashpots in both normal and tangential direction. The perpendicular dashpots to the boundary absorb the p-waves, whereas the dashpots tangential to the boundary absorb the s-waves. In these models, no displacement constraints are used. Therefore, the horizontal at rest earth pressure is applied at the boundary nodes situated at the both sides of the foundation domain. This is done by recording the reaction forces in the model with fixed boundaries and applying them with opposite sign to the model with absorbing boundaries.

This configuration of boundary conditions has no fixed point in $x$-direction. Because the dash pots only provide resistance to high velocity motions the model is very sensitive to low frequency components of the motion. The slightest imbalance in acceleration causes the entire model to move as a rigid body in $x$-direction (Preisig, 2002). To avoid this, the node at the center of the base is fully fixed in the subsequent analyses.

Keeping the center node at the base of the foundation as fixed, two varieties of boundary conditions were tested. In the first case, all the bottom nodes were provided with rollers allowing only horizontal movements as shown in Figure 7 (b) and second, the boundary nodes at the bottom of the base were fitted with dashpots as shown in Figure 7 (c). However, in both these cases, the side nodes of the foundation domains were attached to dashpots in both normal and tangential directions. A detailed description of the use of dashpots can be found in the works by Preisig (2002).

Table 1 Comparison of maximum horizontal crest displacements

\begin{tabular}{|c|c|c|c|c|c|c|}
\hline \multicolumn{2}{|c|}{$\begin{array}{l}\text { Horizontal crest } \\
\text { displacements } \\
\text { (mm) }\end{array}$} & $\begin{array}{c}\text { Yazdchi (et al, } \\
\text { 1999) }\end{array}$ & $\begin{array}{l}\text { Staggered method } \\
\text { with boundary } \\
\text { conditions as per } \\
\text { Figure } 7 \text { (b) }\end{array}$ & $\begin{array}{c}\% \text { of } \\
\text { deviation }\end{array}$ & $\begin{array}{l}\text { Staggered method } \\
\text { with boundary } \\
\text { conditions as per } \\
\text { Figure } 7 \text { (c) }\end{array}$ & $\begin{array}{c}\% \text { of } \\
\text { deviation }\end{array}$ \\
\hline \multirow{8}{*}{$\begin{array}{l}\text { Impedance ratio } \\
\qquad\left(E_{f} / E_{d}\right)\end{array}$} & \multirow{2}{*}{0.5} & 6.89 & 6.99 & 1.45 & 7.14 & 3.63 \\
\hline & & -7.53 & -7.20 & 4.38 & $\begin{array}{l}-7.38 \\
\end{array}$ & 1.99 \\
\hline & \multirow{2}{*}{1.0} & 4.38 & 4.72 & 7.76 & 4.94 & 12.79 \\
\hline & & -4.41 & -4.28 & 2.95 & -4.69 & 6.35 \\
\hline & \multirow{2}{*}{2.0} & 4.27 & 4.11 & 3.74 & 4.36 & 2.11 \\
\hline & & -3.85 & -3.97 & 3.12 & -3.99 & 3.64 \\
\hline & \multirow{2}{*}{4.0} & 4.11 & 3.90 & 5.11 & 3.95 & 3.89 \\
\hline & & -3.70 & -3.57 & 3.51 & -3.47 & 6.22 \\
\hline
\end{tabular}

The nodes at the truncated boundary as well as at the bottom are provided with dashpots in both the horizontal and tangential direction. In spite of these anomalies with the original solution by Yazdchi et al. (1999), the proposed model yields a similar displacement pattern of the horizontal displacement at the crest node though the magnitudes differed. In the present work, the hydrodynamic pressures at the upstream face of the dam are calculated from eq. (6). These hydrodynamic forces are applied on the upstream face of the dam. Table 1 shows the comparison between the results of Yazdchi et al (1999) and that of the proposed method for different $E_{f} / E_{d}$ (impedance ratio) ratios. The maximum crest displacement of the dam under seismic excitation by both the method has been tabulated in Table 1 for comparison. The obtained displacements by the proposed interaction scheme are in very close agreement with the results obtained by Yazdchi et al (1999). The slight discrepancy between the two results might be due to i) use of different methods and numerical tools for the solution of the coupled system and ii) different mesh sizes considered for the problem. Moreover it is observed that the results from the model where bottom nodes were provided with rollers are closer to that of Yazdchi et al (1999) compared to the results obtained from the model where the bottom nodes are also attached to dashpots. Therefore, in subsequent calculations, the bottom nodes of the foundation domain were provided with rollers except the center node which will remain to be fully fixed to prevent any type of rigid body translation. 
3.3 Response of Koyna gravity dam-nonlinear foundation system: The seismic response of Koyna dam has been investigated considering the interaction behavior of a linear concrete dam and a nonlinear, elastic foundation subjected to Koyna earthquake (1967) acceleration. The foundation material is assumed to be of hard rock. The width and the depth of the foundation are assumed to be $250.0 \mathrm{~m}$ and $100.0 \mathrm{~m}$ respectively. The geometry of the dam-foundation system chosen for the analysis purpose is shown in Figure 8. The material properties of the dam are as follows:

The Young's modulus of dam body is assumed to be $3.15 \mathrm{e}+10 \mathrm{~N} / \mathrm{m}^{2}$. The Poisson's ratio is taken to be 0.20 and the mass density is assumed as $2415.816 \mathrm{~kg} / \mathrm{m}^{3}$.

The Young's modulus of the foundation varied in each time step according to the relationship expressed in eq. (3) as proposed by Duncan-Chang model (1970). In this way, the nonlinear nature of the stress-strain behavior of the foundation material is simulated. The material properties of the foundation are as follows:

The Young's modulus of foundation rock is considered to be $1.75 \mathrm{e}+10 \mathrm{~N} / \mathrm{m}^{2}$. The Poisson's ratio is assumed as 0.2 . The mass density of the foundation material is assumed to be $1800.0 \mathrm{~kg} / \mathrm{m}^{3}$. The cohesion of the rock foundation is considered to be $547.22 \mathrm{e}+4 \mathrm{~N} / \mathrm{m}^{2}$ and angle of internal friction is taken as $61.93^{0}$.

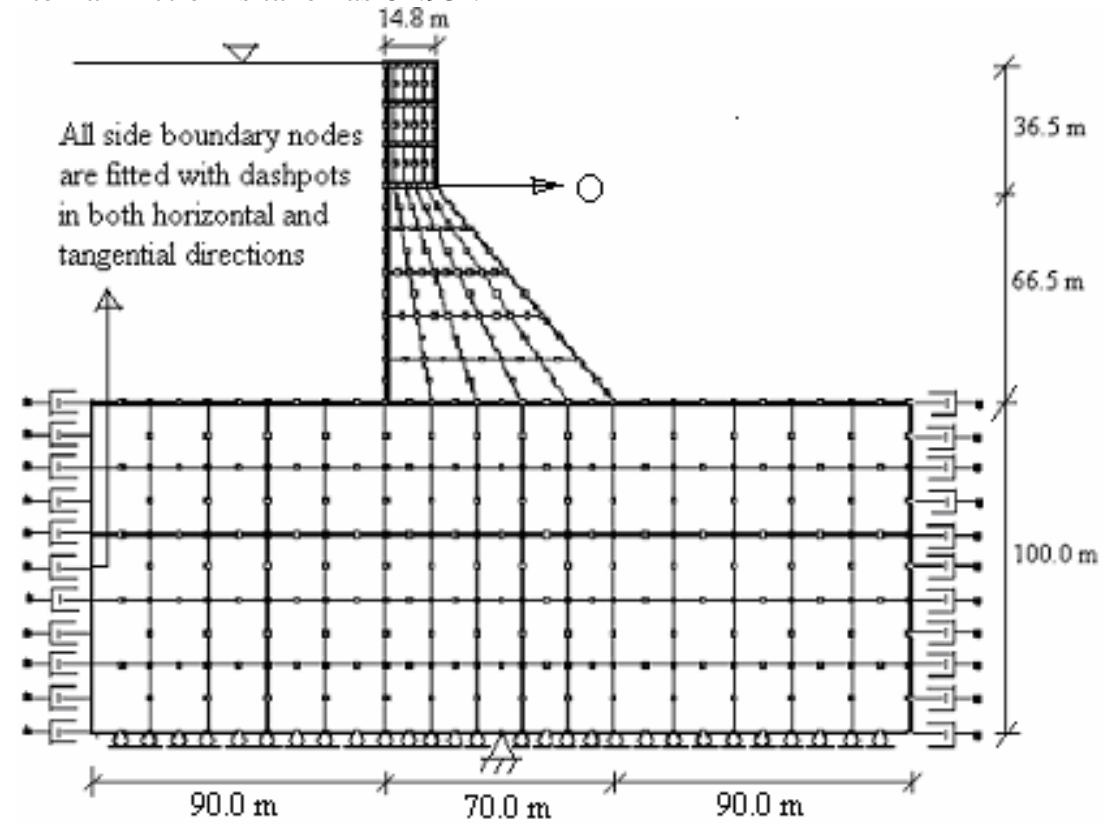

Figure 8. The geometry and boundary condition of the Koyna dam-foundation system

The cohesion $(c)$ and the angle of internal friction $(\phi)$ were assumed according to the values considered by Owen and Hinton (1980). Similar values for these parameters (for the foundation domain) were assumed while analyzing the response of a concrete gravity dam based on a rocky foundation.

In order to arrive at an optimum mesh grading for this particular problem, the response of the dam and the foundation domain under the action of a given load was found out. For the dam part, a $1000 \mathrm{kN}$ load was applied horizontally at the top and the horizontal displacement of the crest node was observed. Also, the time periods of the dam for the first two modes were noted. It was observed that the results converged sufficiently for a mesh grading of $8 \times 5$. For the foundation domain, similar analyses were carried out applying a vertical point load of $1000 \mathrm{kN}$ in the downward direction at the middle of the surface of the foundation. The displacement at the point of application of the load and the time periods for the first two modes were observed. It was observed that the results converged for a mesh size of $15 \times 5$ with sufficient degree of accuracy. However, the results for this analysis are not provided here as they are similar in nature with those of the previous analysis of dam-foundation prototype solved by Yazdchi et al (1999). 


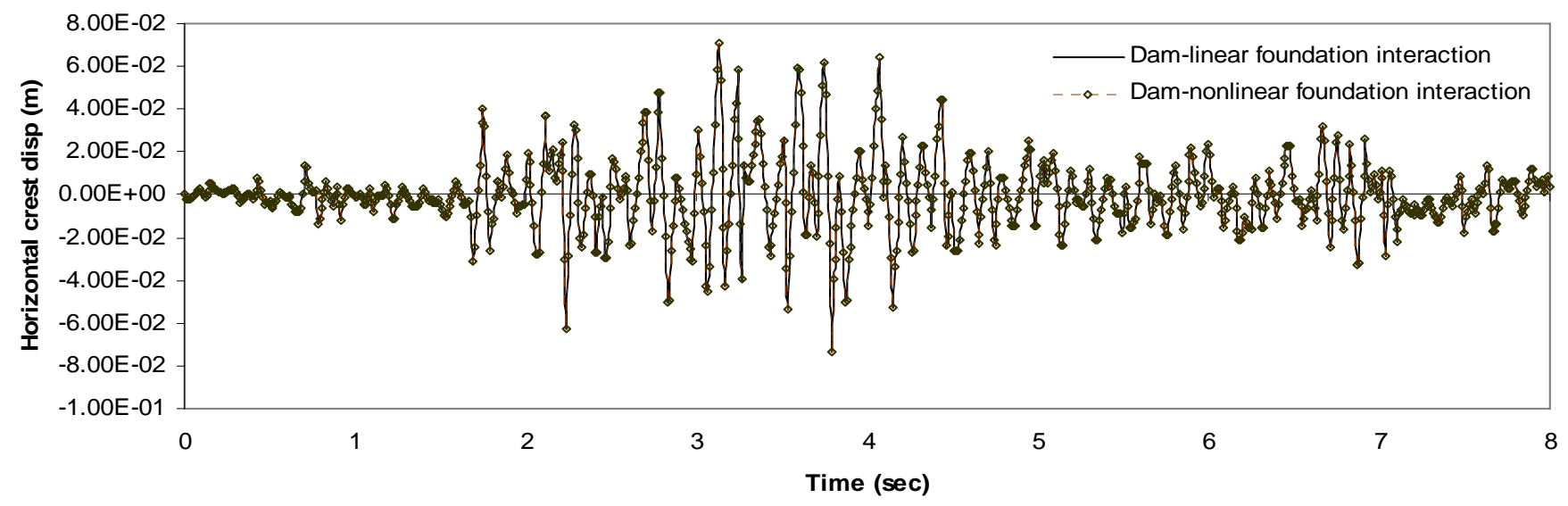

Figure 9. Comparison of horizontal crest displacements between dam and linear/nonlinear foundation

$$
\left(C=547.22 \mathrm{e}+4 \mathrm{~N} / \mathrm{m}^{2} \text { and } \phi=61.93^{\circ}\right)
$$

Figure 9 shows the comparison between the results of the interaction analyses if the material nonlinearity of the foundation material is considered. It is seen that with the already stated cohesion value of $547.22 \mathrm{e}+4 \mathrm{~N} / \mathrm{m}^{2}$ and angle of internal friction of $61.93^{\circ}$, there is almost little or no variation between the magnitudes of horizontal crest displacement between linear and nonlinear case. If the foundation material is considered to be linear, elastic, then the maximum and minimum horizontal crest displacements are found out to be $7.04 \mathrm{~cm}$ and $-7.21 \mathrm{~cm}$ respectively. If the foundation material is modeled with Duncan-Chang model with the above-mentioned material properties, then the interaction analysis produces maximum and minimum displacements of $7.07 \mathrm{~cm}$ and $-7.26 \mathrm{~cm}$ respectively. Therefore a variation of $0.43 \%$ and $0.69 \%$ were observed between the results of two cases. Because of such little variations, the material properties of the foundation were changed in order to exaggerate the effect of nonlinearity of the foundation material. For further analyses, the cohesion and the angle of internal friction of the foundation material were considered to be $150.0 \mathrm{e}+4 \mathrm{~N} / \mathrm{m}^{2}$ and $40.0^{0}$ respectively.



Figure 10. Horizontal crest displacements vs. time for dam and linear/nonlinear foundation interaction

$$
\left(C=150.0 \mathrm{e}+4 \mathrm{~N} / \mathrm{m}^{2} \text { and } \phi=40.0^{\circ}\right)
$$

Figure 10 shows the comparison of interaction analyses with dam and linear/nonlinear foundation material with changed material properties. In this case, the interaction analysis with nonlinear material properties produced maximum and minimum crest displacements as $8.18 \mathrm{~cm}$ and $-8.02 \mathrm{~cm}$. Therefore, a variation of $16.19 \%$ and $11.23 \%$ were observed with the results of linear foundation material properties. Figure 11 shows the variation of major principal stress vs time for dam and linear/nonlinear foundation interaction analyses plotted at point ' $\mathrm{O}$ ' as shown in Figure 8. The maximum values were observed to be $15.30 \mathrm{MPa}$ and 16.45 MPa respectively for interaction analyses of dam with linear and nonlinear foundation material. For nonlinear analysis, the major principal stress was observed to rise by $7.52 \%$ compared to the case where foundation material was assumed to be linear, elastic. Figure 12 shows the variation of minor principal stress vs time for dam and linear/nonlinear foundation material obtained at node ' $\mathrm{O}$ ' as shown in Figure 8. The maximum values of principal stress were observed to be $-14.50 \mathrm{MPa}$ and $-15.90 \mathrm{MPa}$ 
respectively for interaction analyses of dam and foundation with both linear and nonlinear material properties. Therefore, for nonlinear case, an increase of $9.66 \%$ in the magnitude of minor principal stress was noticed.

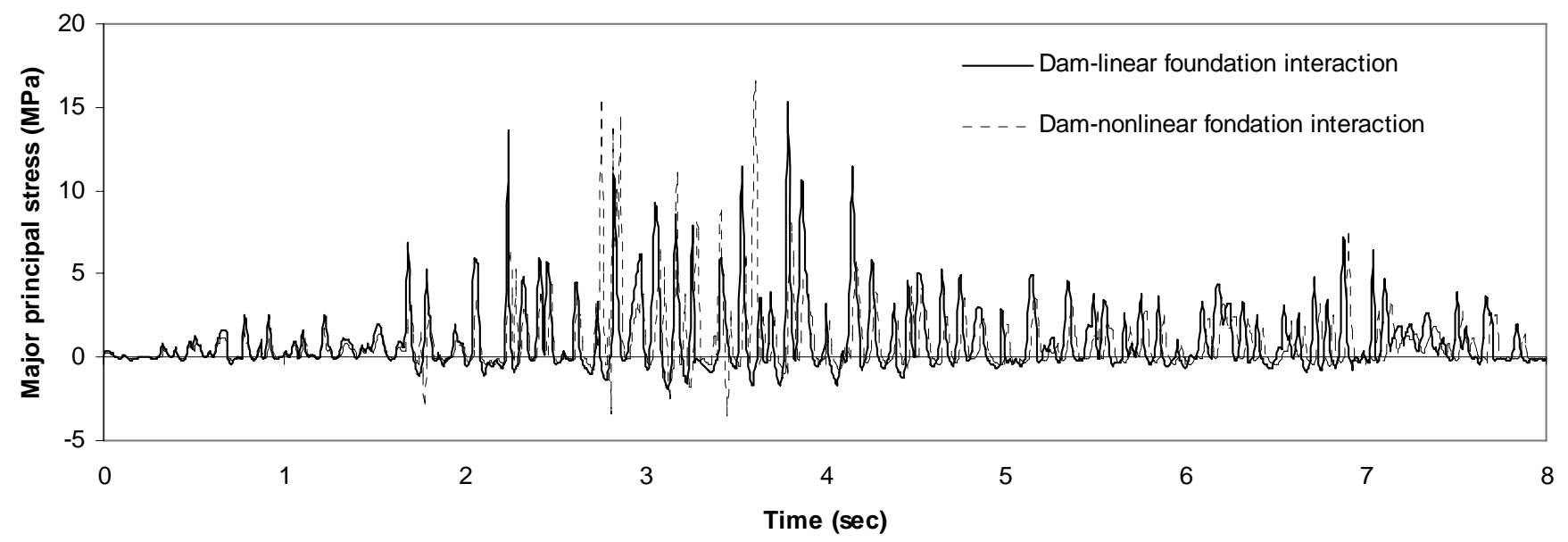

Figure 11. Major principal stress vs time for dam and linear/nonlinear foundation interaction at point ' $O$ ' $\left(C=150.0 \mathrm{e}+4 \mathrm{~N} / \mathrm{m}^{2}\right.$ and $\left.\phi=40.0^{\circ}\right)$

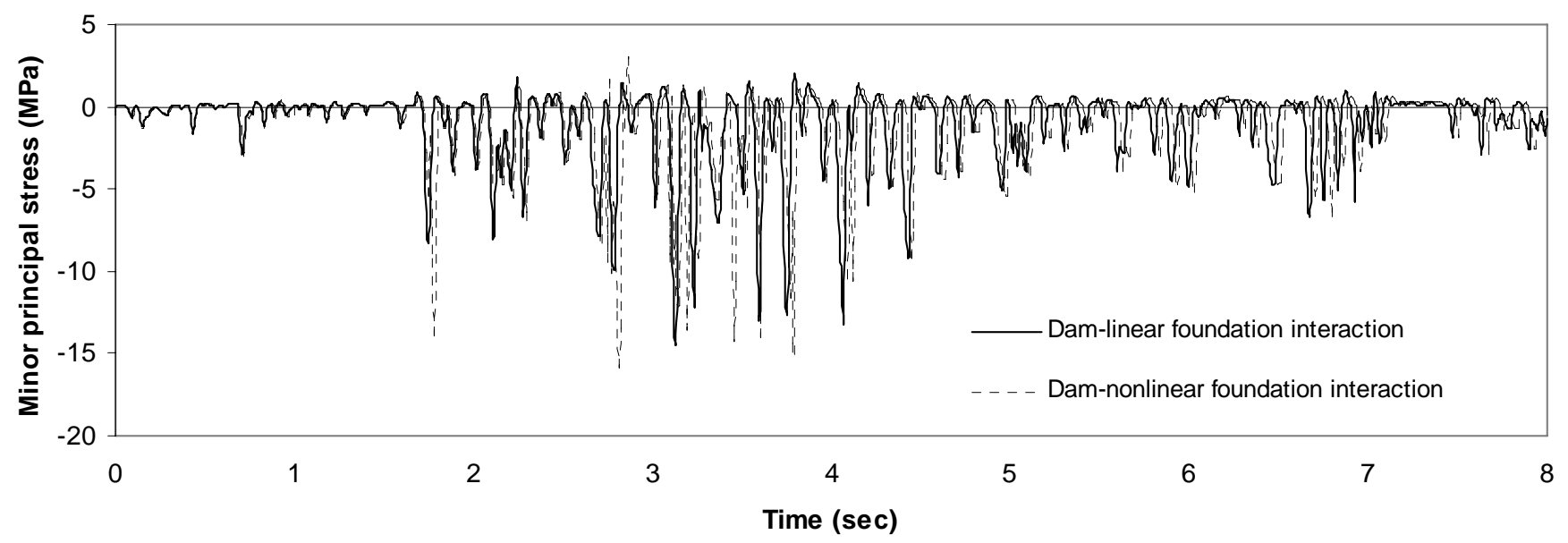

Figure 12. Minor principal stress vs time for dam and linear/nonlinear foundation interaction at point ' $O$ '

$$
\left(C=150.0 \mathrm{e}+4 \mathrm{~N} / \mathrm{m}^{2} \text { and } \phi=40.0^{\circ}\right)
$$

\section{Conclusions}

The present paper illustrates a simple iterative method for the dynamic analysis of nonlinear soil-structure interaction problems considering a concrete gravity dam situated on rock foundation. The two individual systems, i.e., dam and foundation are solved separately and the interaction effects are incorporated through an iterative procedure. The iterative method breaks the whole system into two separate sub-systems. As a result, the corresponding matrices are much smaller and consequently takes lesser time for solution of individual system. The proposed method is validated from the literature which shows the accuracy of the developed algorithm. The Koyna dam-foundation system has been analysed against Koyna earthquake acceleration. The effect of hydrodynamic pressure on the dam body has been included in the dynamic analysis of dam-foundation coupled system. The nonlinear nature of the foundation material is simulated by considering Duncan-Chang model. It is clear that consideration of nonlinear material behavior of the foundation domain produces higher response (displacements and stresses) compared to the case when the foundation is assumed to be linear and elastic. Therefore, when the structure is massive like dam and is being excited by seismic forces, it is always advisable to carry out non-linear foundation-dam interaction analysis to obtain accurate behavior of the coupled system. 


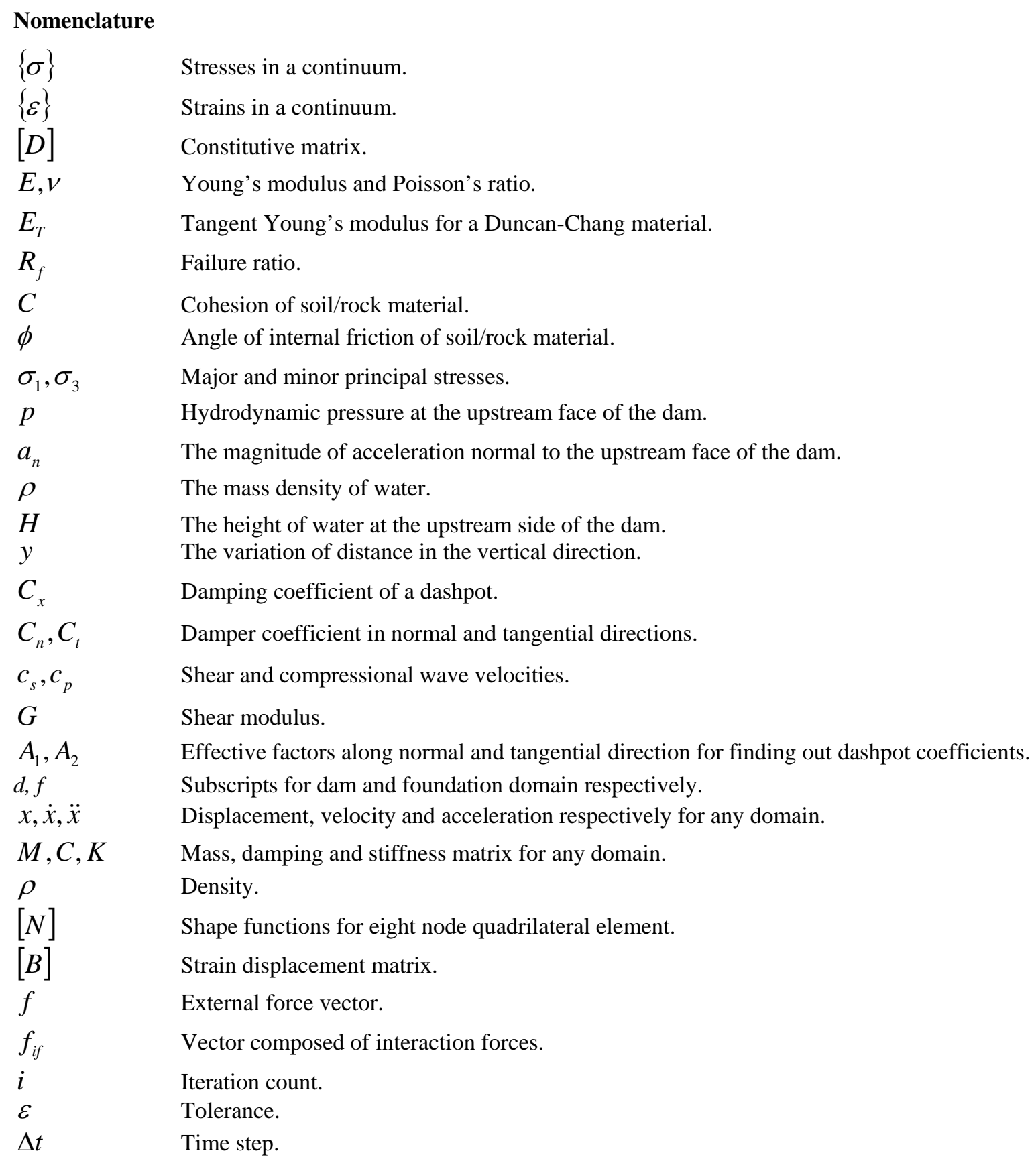

\section{References}

Abouseeda H. and Dakoulas, P., 1998. Nonlinear dynamic earth dam-foundation interaction using a FE-BE method, Earthquake Engineering and Structural Dynamics, Vol. 27, pp. 917-936.

Bettes P., 1977. Infinite elements, International Journal Numerical Methods for Engineering, Vol. 11, pp. 53-64.

Bose S. K. and Das S. C., 1997. Nonlinear finite element analysis of stresses and deformations beneath rigid footings, Computers \& Structures, Vol. 62, No. 3, pp. 487-492.

Degroote J., Haelterman R., Annerel S., Bruggeman P. and Vierendeels J., 2010. Performance of partitioned procedure in fluidstructure interaction, Computers \& Structures, Vol. 88, No. 7, pp. 446-457.

Duncan J. M. and Chang C. Y., 1970. Non-linear analysis of stress and strain in soils, Journal of Soil Mechanics and Foundation Engineering Division, ASCE, Vol. 96(SM5), pp. 1629-1634.

Estorff O. Von. and Kausel E., 1989. Coupling of boundary and finite elements for soil-structure interaction problems, Earthquake Engineering and Structural Dynamics, Vol. 18, pp.1065-1075. 
Estorff O. Von. and Firuziaan M., 2000. Coupled BEM/FEM approach for nonlinear soil-structure interaction, Engineering Analysis with Boundary Elements, Vol. 24, No. 10, pp.715-725.

Felippa C.A. and Park K.C., 1980. Staggered transient analysis procedures for coupled mechanical systems: formulation, Computer Methods in Applied Mechanics and Engineering, Vol. 24, pp. 61-111.

Jahromi H. Z., Izzuddin B. A. and Zdravkovic L., 2007. Partitioned analysis of nonlinear soil-structure interaction using iterative coupling, Interaction and Multiscale Mechanics, Vol. 1, No. 1, pp. 33-51.

Jahromi H. Z., Izzuddin B. A. and Zdravkovic L., 2009. A domain decomposition approach for coupled modeling nonlinear soilstructure interaction, Computer Methods in Applied Mechanics and Engineering, Vol. 198, No. 33, pp. 2738-2749.

Khalili N., Valiappan S., Tabatabaie Yazdi. J. and Yazdchi M., 1997. 1D infinite element for dynamic problems in saturated media, Communications in Numerical. Methods in Engineering, Vol. 13, pp. 727-738.

Lysmer J. and Kuhlemeyer R. L., 1969. Finite dynamic model for infinite media, Journal of Engineering Mechanics Division, ASCE, Vol. 95 (EM4), pp. 859-877.

Maity D. and Bhattacharyya S.K., 2003. A parametric study on fluid structure interaction problems. Journal of Sound and Vibration, Vol. 263, pp. 917-935.

Owen D. R. J. and Hinton E., 1980. Finite Elements in Plasticity: Theory and Practice, Penshaw Press Limited, Swansea U. K., Chapter 10.

Preisig M., 2002. Nonlinear finite element analysis of dynamic soil-foundation-structure interaction, Dissertation submitted for Master of Science in Civil Engineering, University of California, Davis. Swiss Federal Institute of Technology, Lausanne.

Valiappan S. and Zhao C., 1992. Dynamic response of concrete gravity dams including dam-water-foundation interaction, International Journal Numerical Methods for Engineering, Vol. 16, pp. 79-99.

Westergaard H. M., 1933. Water pressures on dams during earthquakes, Trans. ASCE, Vol. 98, pp. 418-472.

White W., Valliappan S. and Lee I. K., 1977. Unified boundary for finite dynamic models, Journal of Engineering Mechanics Division, ASCE, Vol. 103, pp. 949-964.

Wolf J.P., 1985. Dynamic soil-structure interaction, Prentice Hall, Englewood Cliffs, NJ.

Wolf J. P and Obernhuber P., 1985. Nonlinear soil-structure interaction analysis using Green's function of soil in the time domain, Earthquake Engineering and Structural Dynamics, Vol. 13, pp. 213-223.

Wolf J. P and Darbre G. R., 1986. Nonlinear soil-structure interaction analysis based on the boundary element method in time domain with application to embedded foundation, Earthquake Engineering and Structural Dynamics, Vol. 13, pp. 83-100.

Wolf J.P. and Song C., 1996. Finite element modeling of unbounded media, Wiley, New York.

Yazdchi M., Khalili N. and Valliappan S., 1999. Dynamic soil-structure interaction analysis via coupled finite element-boundary element method. Soil Dynamics and Earthquake Engineering, Vol. 18, pp. 499-517.

Zhao C. and Liu T., 2003. Non-reflecting artificial boundaries for transient scalar wave propagation in a two dimensional infinite homogenous layer, International Journal for Numerical Methods in Engineering, Vol. 58, pp. 1435-1456.

Zienkiewicz O.C., Kelly D.W. and Bettes P., 1977. The coupling of the finite element methods and boundary solution procedures, International Journal for Numerical Methods in Engineering, Vol. 11, pp. 355-377.

\section{Biographical notes}

Avijit Burman is a Lecturer, Department of Civil Engineering, Birla Institute of Technology, Ranchi (India). He is engaged in teaching and research activities since the last 6 years. His field of specialization is soil-structure interaction.

Dr. Damodar Maity is an Associate Professor in the Department of Civil Engineering, Indian Institute of Technology, Kharagpur, India. His research interest is in the field of computational mechanics, fluid-structure interaction, soil-structure interaction. He has published several research papers in many international and national journals. He is author of a book, titled, "Computer Method of Framed Structures”.

Dr. S. Sreedeep is working as an Assistant Professor in Civil Engineering Department, Indian Institute of Technology, Guwahati, India. He has done his research in the field of environmental geotechnical engineering. He has published several research papers in many international and national journals.

Received December 2009

Accepted April 2010

Final acceptance in revised form May 2010 